\title{
Mechanical Behavior at High Temperature of a Centrifugally-cast Stainless Steel Tube
}

\author{
J. A. JIMÉNEZ, O. A. RUANO, R. ALLENDE ${ }^{1)}$ and M. CARsí
}

Department of Physical Metallurgy, Centro Nacional de Investigaciones Metalúrgicas, C.S.I.C., Av. Gregorio del Amo 8,28040 Madrid, Spain. $\quad$ 1) Productos Tubulares S. A., Carretera de Ugarte-Galindo s/n, 48510 Valle del Trápaga, Vizcaya, Spain.

(Received on December 1, 1997; accepted in final form on April 3, 1998)

\begin{abstract}
Mechanical properties at high temperature of a centrifugally-cast stainless steel tube have been investigated by torsion tests. Microstructure and composition have been determined in different positions along the tube radius. Differences in the microstructure related to different solidification rates have been observed between the interior and exterior zones. Garofalo's equation has been calculated considering the results of torsion tests of samples obtained from both zones. A similar value for the activation energy and hyperbolic sinus exponent have been observed in both cases. However, a change in ductility and fracture stress between both zones has been found at temperatures above $1100^{\circ} \mathrm{C}$.
\end{abstract}

KEY WORDS: stainless steel tubes; centrifugally-cast; thermomechanical behaviour.

\section{Introduction}

The $\mathrm{Fe}-\mathrm{Cr}-\mathrm{Ni}$ alloys have been widely used over the last half-century as special materials for construction of a great variety of applications demanding high corrosion resistance. For this reason, the mechanical properties and forming behavior of the $\mathrm{Cr}-\mathrm{Ni}$ stainless have been widely studied. ${ }^{1,2)}$ These steels exhibits good combination of ductility and toughness even at high-strength levels. These mechanical characteristics are imparted by the austenitic microstructure stabilized at room temperature with a minimum of $8 \% \mathrm{Ni}$.

Semifinished tube rounds products are hollow products made either by piercing rounds or by rolling and welding strip. ${ }^{3)}$ An entirely different approach to tube production uses horizontal centrifugal-casting. ${ }^{4)}$ In this case, well-refined molten steel is poured into a rotating metal mold with flux.

As mechanical properties of materials can be drastically changed by the processing routes, the application of centrifugal-casting technology could introduce different values of the warm-forming thermomechanical parameters to those used conventionally. In this sense, this work is aimed to determine and discuss the possible influence of centrifugal-casting on the hot-deformation resistance, and, in this case, to quantify these differences. Besides, emphasis will be placed on determining the microstructural differences developed in the radial direction. These differences may drive to a gradient in the mechanical properties like toughness or wear resistance. Therefore, this study will contribute to provide, to high performance steel tube makers, a certain degree of security about the uniformity in the response to the different steps included in thermomechanical processing of a centrifugally-cast steel.

\section{Experimental Procedure}

A commercial centrifugal-cast 18-8 stainless steel was supplied as a tube of $20 \mathrm{~mm}$ in wall thickness. The chemical composition of this tube in both zones, interior and exterior, was determined by Inductively Coupled Plasma, except for carbon and sulfur that were determined by infrared absorption after combustion in an induction furnace. Within experimental accuracy measured, the same chemical analytical results were obtained in both zones: $0.050 \mathrm{C}-0.49 \mathrm{Si}-1.47 \mathrm{Mn}-18.8 \mathrm{Cr}-9.81 \mathrm{Ni}-$ $0.016 \mathrm{~S}-0.014 \mathrm{P}$ (mass in \%).

Microstructural characterization of the as-received alloy and after deformation was performed by optical microscopy. Metallographic preparation included mounting samples in baquelite and polishing by the conventional method. The microstructure was revealed by electrolytically etching at room temperature in a $10 \% \mathrm{NaOH}$ solution.

Elevated temperature mechanical properties were characterized by tensile and torsion tests performed at equivalent strain rates ranging from 1 to $20 \mathrm{~s}^{-1}$ in the temperature range from 900 to $1250^{\circ} \mathrm{C}$. In order to study the influence of the stage of segregation in the mechanical properties, torsion tests were carried out with samples taken from two zones of the tube, interior and exterior. Torsion samples with a circular cross section of $6 \mathrm{~mm}$ in diameter and a gauge length of $17 \mathrm{~mm}$ were machined in the direction parallel to the tube axis. The samples were introduced in a silica tube with a helium inlet to ensure protection against oxidation and to minimize adiabatic heating. The equivalent true flow stress and 


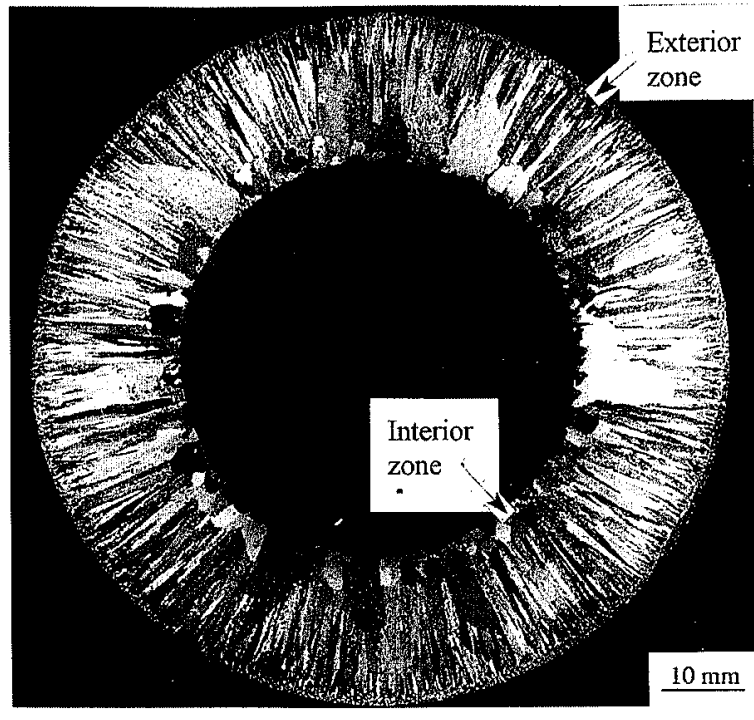

Fig. 1. Macrograph of a cross section of the centrifugal-cast tube.

strain were calculated from the experimental torquenumber of revolutions curves using Fields and Backofen relation. ${ }^{5)}$

\section{Results and Discussion}

\subsection{Microstructure}

A macrograph of a cross section of the centrifugalcast tube is given in Fig. 1. The macrograph illustrates the presence of three basic solidification zones: a layer of about $4 \mathrm{~mm}$ consisting of fine and equiaxic grains in the neighborhood of the external wall (exterior zone); an adjoined layer with a structure of columnar grains oriented parallel to the radial direction (columnar zone); and an innermost layer of about $8-10 \mathrm{~mm}$ with coarse and equiaxic grains (interior zone). These different microstructures have been related with differences in the solidification conditions between the exterior and interior zones. When the molten steel is poured into a rotating metal mold, the mold wall (at much lower temperature than the melt) rapidly cools the layer of the liquid in contact with it. Therefore, a considerable magnitude of thermal and constitutional supercooling results. Heterogeneous nucleation will occur relatively rapid, and a fine and equiaxic layer of crystal will be obtained. As soon as the nucleation starts, the release of the latent heat of fusion determines an inversion in the temperature ahead of the crystal formed in the chill zone. Instead of the temperature rising ahead of the interface it now falls. This decreasing temperature of the liquid in the advance of the interface promotes dendritic growth, and with it a corresponding development of a preferred orientation. A columnar zone begins to form with a dendritic growth direction in each columnar grain parallel to the heat flow direction. The growth of these crystals stops when they meet the grains growing from the interior zone. As the interior zone is in contact with the air, the solidification rate is much lower than in the exterior zone, and the grains in the corresponding equiaxed layer are coarser.

On the other hand, a metallographic analysis at higher

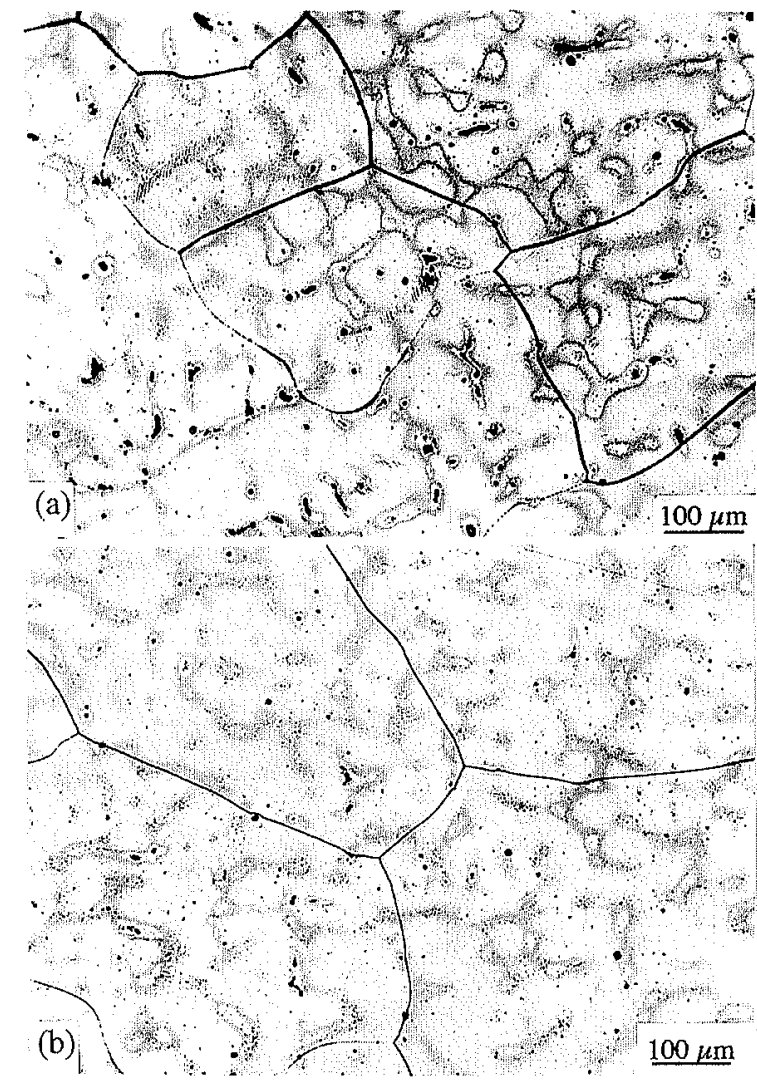

Fig. 2. As-cast microstructure showing a dendritic microstructure in the interior (a) and exterior (b) zones of the tube.

magnification confirmed the presence of a dendritic microstructure, as shown in Figs. 2(a) and 2(b), for the interior and exterior zones of the tube, respectively. As the driving force for dendritic growth in alloys is the constitutional supercooling, the regions between the dendritic arms are enriched in solute concentration. The dendritic segregation present in the as cast material can be eliminated by a homogenization heat treatment.

A microstructural refinement is observed after torsion testing. Dynamic recrystallization starts after a critical strain and, in the course of deformation, a steady state region, characterized by a constant value of stress, is reached. At this point, there is a stable equilibrium between strain hardening and dynamic recrystallization plus recovery and a fine and stable microstructure is developed, as shown in Figs. 3(a) and 3(b). These figures show the microstructure obtained after torsion testing $(\varepsilon=2)$ at $1100^{\circ} \mathrm{C}$, for the interior and exterior zones, respectively. As can be seen, both microstructures are very similar.

\subsection{Mechanical Behavior}

No difference on mechanical behavior was found between the exterior and the interior zones of the tube at room temperature. The same results from hardness and tensile tests were obtained with samples extracted from both zones.

The mechanical behavior at high temperature was investigated by means of torsion tests, from which the stress and temperature dependencies of the strain rate 


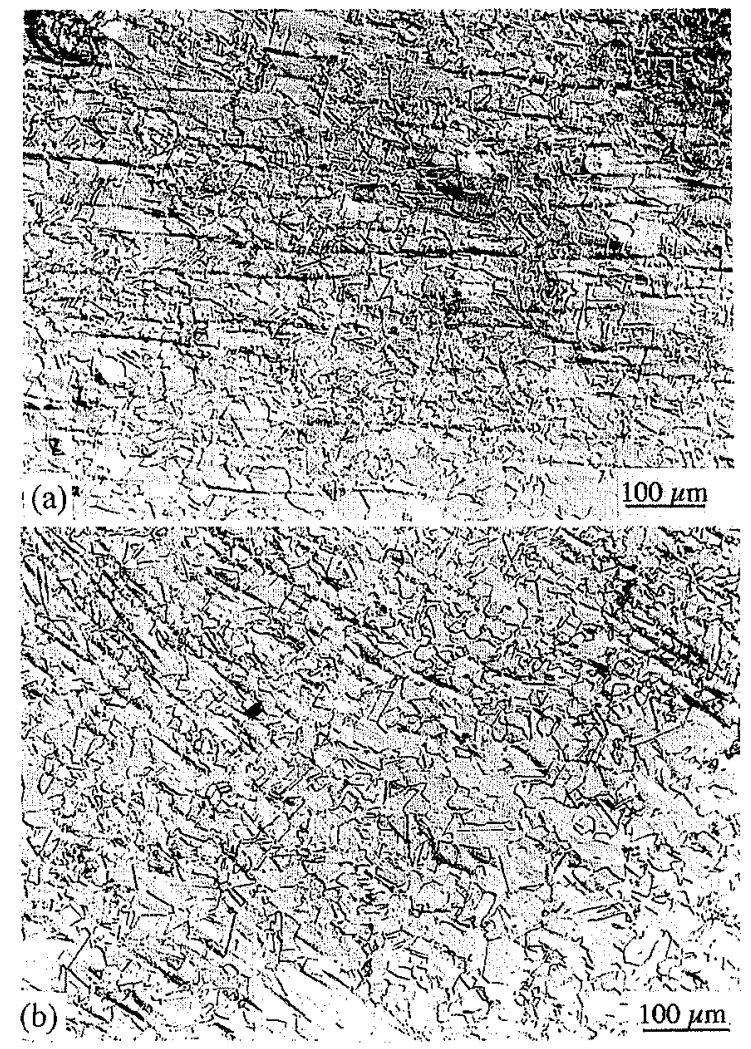

Fig. 3. Microstructure transformed after deformation at $I 100^{\circ} \mathrm{C}$ at an equivalent true strain of $\varepsilon=2$ for the interior (a) and exterior (b) zones.

were obtained. In this study, the equivalent stress and strain rate was calculated at an equivalent true strain of $\varepsilon=2$, at which the steady state region is reached. The results of torsion tests are presented in Figs. 4(a) and 4(b) as plots of the strain-rate, $\dot{\varepsilon}$ (in s ${ }^{-1}$ ), against the Young's modulus compensated flow stress, $\sigma / E$, for the interior and exterior zones of the tube, respectively. The values for the Young's modulus at the various temperatures were taken from data reported for a steel with similar composition. ${ }^{6}$ On these plots, the slope of the curves corresponds to the stress exponent. Figures 4(a) and 4 (b) show a stress exponent of about 5 , which is consistent with the creep rate dependence on the stress deduced in the classical theory when deformation is controlled by a dislocation climb mechanism. ${ }^{7)}$

The results presented in Figs. 4(a) and 4(b) were reanalyzed according to the well-known Garofalo's hyperbolic sine creep relation: ${ }^{81}$

$$
\dot{\varepsilon}=A \exp \left(\frac{-Q_{\mathrm{c}}}{R T}\right)\left(\sinh \alpha \frac{\sigma}{E}\right)^{n}
$$

where $Q_{\mathrm{c}}$ is the activation energy for creep (in $\mathrm{kJ} / \mathrm{mol}$ ), $R$ is the gas constant (in $\mathrm{kJ} / \mathrm{mol}-\mathrm{K}$ ), $T$ is the absolute temperature (in $\mathrm{K}$ ), and $A$ and $\alpha$ are material constants that are obtained from the true stress-true strain data represented in Fig. 4 by means of an algorithm. ${ }^{9,10)}$ This procedure includes an iterative non linear least-squares fitting to Eq. (1) by means of a modified version of the Gauss-Newton method, which use as starting parameters the constant deduced from a linear fit to the equation:
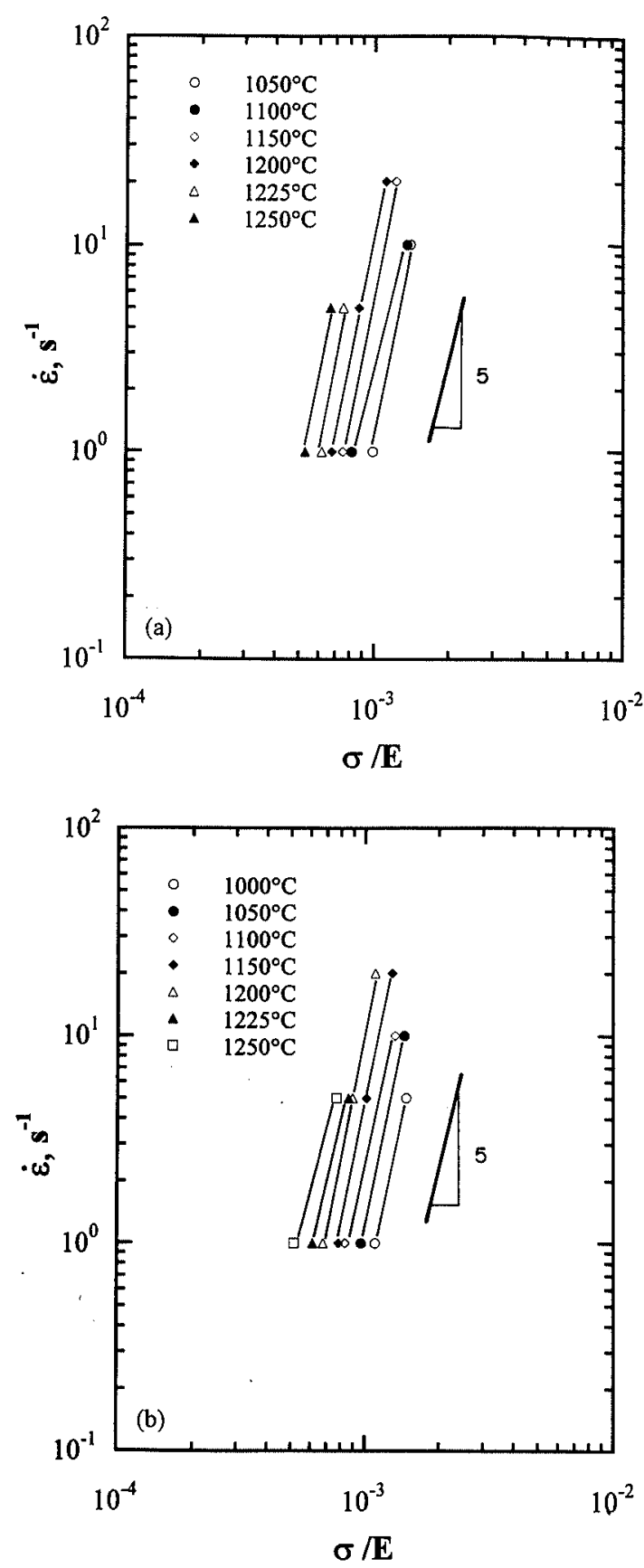

Fig. 4. Strain-rate against the Young's modulus compensated flow stress from torsion test of samples taken from the interior (a) and exterior (b) zones.

$$
\ln \dot{\varepsilon}=\ln A-\frac{Q_{\mathrm{c}}}{R T}+n \ln \left(\sinh \alpha \frac{\sigma}{E}\right)
$$

Equation (1) can be used to generalize the creep behavior of materials at a wide range of stresses, from those at the power law breakdown to those at very low strain rates. For this case, the following Garofalo's fit where obtained for the interior (3) and exterior (4) side of the tube, respectively

$$
\begin{aligned}
& \dot{\varepsilon}=5.58 \times 10^{8} \exp \left(-\frac{232}{R T}\right)\left(\sinh 1072 \frac{\sigma}{E}\right)^{3.97} \\
& \dot{\varepsilon}=5.25 \times 10^{8} \exp \left(-\frac{230}{R T}\right)\left(\sinh 1039 \frac{\sigma}{E}\right)^{4.01}
\end{aligned}
$$




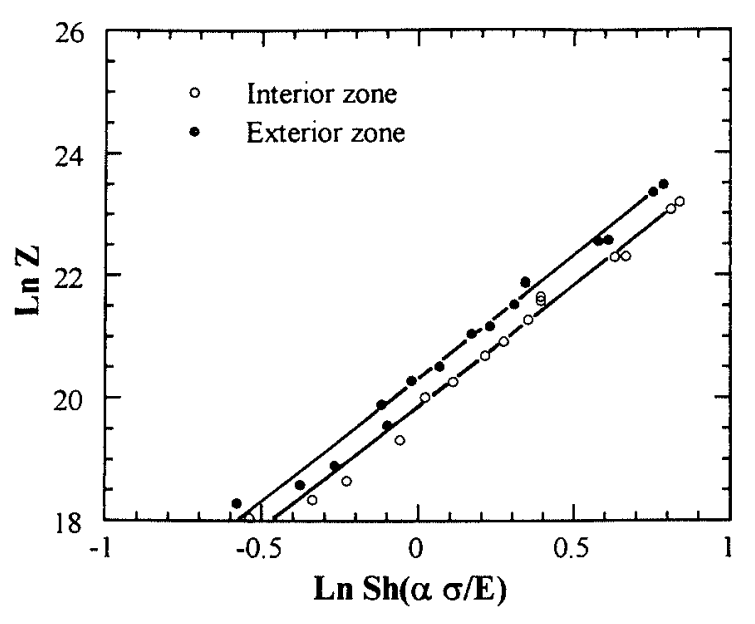

Fig. 5. Zener-Hollomon parameter $(\dot{Z}=\dot{\varepsilon} \exp (Q / R T))$ as a function of the hyperbolic sinus of the Young's modulus compensated stress from torsion tests of samples taken from the interior and exterior zones.

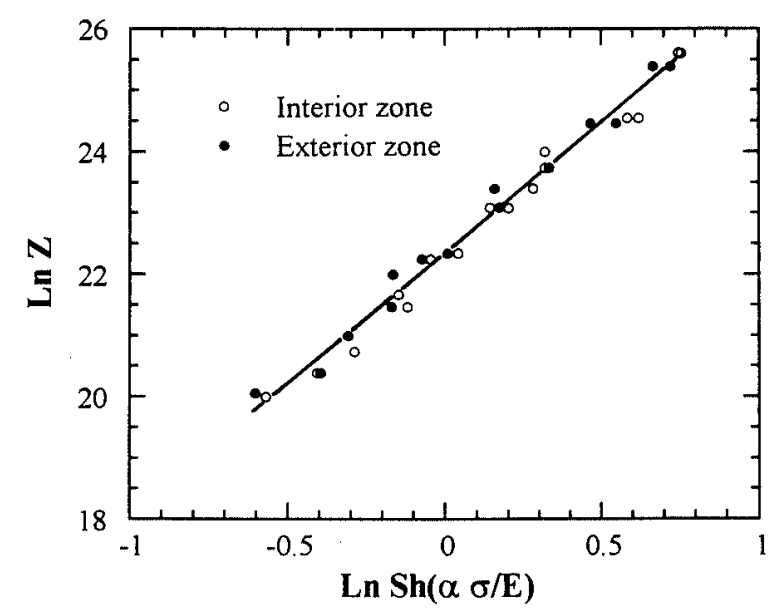

Fig. 6. Single Garofalo's fits from torsion tests of samples taken from the interior and exterior zones.

A value of $F \cong 270$ for the Snedecor's coefficient corresponding to the initial values and a value of $r=0.991$ for the regression coefficient of the final solution were deduced in both regressions. As can be seen, both equations have similar values for the activation energy for plastic deformation and for the hyperbolic sinus exponent. Figure 5 shows the results of the Garofalo's analysis obtained from the torsion tests performed with samples taken from the interior and exterior zones. In this figure, the logarithm of the Zener-Hollomon parameter, $Z=\dot{\varepsilon} \exp \left(-Q_{\mathrm{c}} / R T\right)$, is plotted as a function of the logarithm of $\sinh (\alpha \sigma / E)$. The experimental scatter shown in this figure is sufficiently small to establish that the strain rate is independent of the zone of the tube from which the samples were taken. Therefore, a single fit was considered for both data sets, as shown in Fig. 6. The Garofalo's equation obtained from this figure is given as

$$
\dot{\varepsilon}=2.18 \times 10^{9} \exp \left(-\frac{245}{R T}\right)\left(\sinh 1020 \frac{\sigma}{E}\right)^{4.10}
$$

Again, a value of $r=0.991$ for the regression coefficient and $F \cong 270$ for the Snedecor's coefficient was obtained.
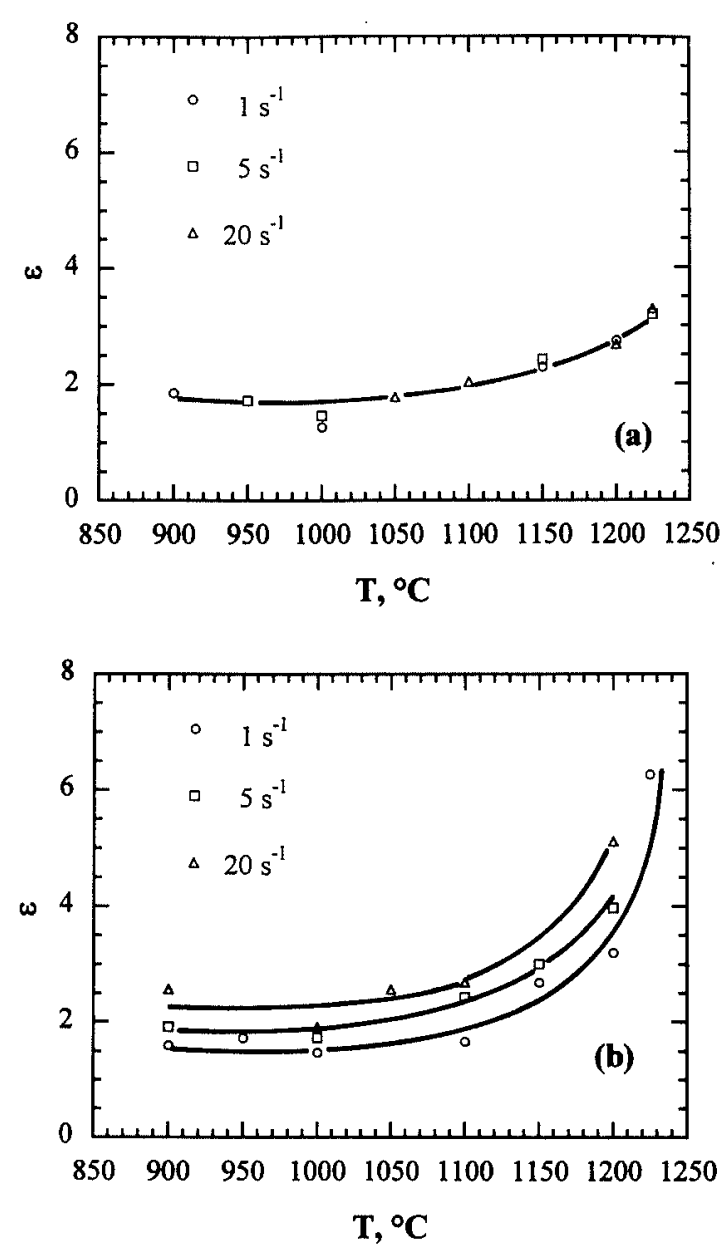

Fig. 7. Equivalent strain as function of testing temperature of samples taken from the interior (a) and exterior (b) zones.

This is an indication that both data sets can be considered as arising from testing the same material. The activation energy for deformation obtained, $245 \mathrm{~kJ} / \mathrm{mol}$, close to those given for the activation energy for lattice diffusion in austenitic stainless steels $(280 \mathrm{~kJ} / \mathrm{mol}),{ }^{11)}$ and basically coincides with that derived from Fig. 4.

Torsion tests to failure in the temperature range from 900 to $1250^{\circ} \mathrm{C}$ at strain rates ranging from 1 to $20 \mathrm{~s}^{-1}$ were conducted as a measurement of the ductility of this alloy. The value for the equivalent strain, $\varepsilon$, was obtained by means of the following expression

$$
\varepsilon=\frac{2 \pi R N}{L \sqrt{3}}
$$

where $L$ is the effective sample length, $R$ is the radius of the sample, and $N$ is the number of turns. For the torsion samples used ( $L=17 \mathrm{~mm}, R=3 \mathrm{~mm})$ this expression is reduced to $\varepsilon=0.64 N$. The equivalent strain is plotted in Figs. 7(a) and 7(b) as a function of the testing temperature, for samples taken form the interior and exterior sides of the tube, respectively. These figures show that the ductility is not a function of the strain rate only for the interior zone. For the exterior zone, the higher the strain rate the higher the ductility. On the other hand, the ductility in both zones increases with rising the temperature because recrystallization starts earlier and proceeds 
faster, so cracks at the stress concentration points are less likely to be initiated.

Although the deformation parameters, deduced from a Garofalo's fit, are the same for the interior and the exterior zones, samples taken from the exterior zone present a higher ductility at temperatures above $1100^{\circ} \mathrm{C}$. This difference in ductility increases with testing temperature. The highest differences in ductility were measured at $1250^{\circ} \mathrm{C}$. At this temperature the elongation to failure for the exterior zone is two times that for the interior zone. The porosity present in the tube, although small, is important for understanding the limited ductility in the interior zone. As reveals by comparing Figs. 2(a) and 2 (b) a higher pore volume fraction is present in the interior zone. One of the basic causes for porosity in metals and alloys is gas evolution during freezing. When the molten steel is pored into a rotating metal mold, bubbles of gas may form in the liquid. As these bubbles have a low density, they will be subjected to a lower centrifugal force than the melt, and then they tend to reach the interior surface. Therefore, the larger pore volume fraction may form at the interior zone.

The heterogeneity of ductility between both zones introduces a risk factor during the processing stages. Therefore, the amount of deformation of each zone has to be considered when designing the processing stages to maintain a uniform quality in the final product along the width of the tube.

\section{Conclusions}

(1) The centrifugally-cast tube presents three basic solidification regions, which are differentiated by size and morphology of the austenitic grains. These different microstructures have been related with differences in the solidification conditions between the exterior and interior zones.

(2) The small difference between the results obtained from the torsion tests performed with samples taken from the interior and exterior zones allows to establish that the strain rate-stress relationship is independent of the position from which the samples were taken.

(3) The analysis of the torsion tests using the Garofalo's hyperbolic sine relation leads to a stress exponent of about 5 and an activation energy for plastic deformation similar to the activation energy for lattice diffusion in austenitic stainless steels. This result is in agreement with a dislocation climb process as the predominant deformation mechanism.

(4) Ductility is not a function of the strain rate only for the interior zone. On the other hand, samples taken from the exterior zone present a higher ductility at temperatures above $1100^{\circ} \mathrm{C}$ related with a lower pore volume fraction. This heterogeneity of ductility could introduce a risk factor in the processing stages.

\section{Acknowledgement}

The authors gratefully acknowledge the support of the Comisión Interministerial de Ciencia y Tecnología (CICYT) under Grant MAT 97/0700.

\section{REFERENCES}

1) D. Peckner and I. M. Bernstein: Handbook of Stainless Steels, McGrawhill Inc., New York, (1977).

2) Chromium-nickel Stainless Steel Data, The International Nickel Company, Inc., New York, (1963)

3) H. F. Rase: Piping Design for Process Plants, John Wiley \& Sons, Inc., New York, (1963)

4) S. Schwaigerer: Rohrleilungen, Springer-Verlag, Berlin, (1967).

5) D. S. Fields and W. A. Backofen: Ant. Soc. Test. Mater. Proc., 57 (1957), 1259

6) C. W. Andrews: Met. Prog., 58 (1950), 85.

7) J. Weertman: Trans. Am. Soc: Met., 61 (1968), 681.

8) F. Garofalo: Trans. Metall. Soc. AIME, 227 (1963), 351.

9) I. Rieiro: Ph. D. Thesis, Universidad Complutense de Madrid, Madrid, Spain, (1997).

10) I. Rieiro, M. Carsi and F. Peñalba: Rev. Metal. Madrid, 32 (1996), No. 5,321 .

11) B. Million, J. Ruzickova and J. Vrestal: Mater. Sci. Eng., 72 (1985), 85 